This is the author's final, peer-reviewed manuscript as accepted for publication. The publisher-formatted version may be available through the publisher's web site or your institution's library.

\title{
Position of the Academy of Nutrition and Dietetics: nutrition and lifestyle for a healthy pregnancy outcome
}

Sandra B. Procter, Christina G. Campbell (Lead Author)

\section{How to cite this manuscript}

If you make reference to this version of the manuscript, use the following information:

Procter, S. B., Campbell, C. G. (2014). Position of the Academy of Nutrition and Dietetics: Nutrition and lifestyle for a healthy pregnancy outcome. Retrieved from http://krex.ksu.edu

\section{Published Version Information}

Citation: Procter, S. B., Campbell, C. G. (2014). Position of the Academy of Nutrition and Dietetics: Nutrition and lifestyle for a healthy pregnancy outcome. Journal of the Academy of Nutrition and Dietetics, 114(7), 1099-1103.

Copyright: (c) 2014 by the Academy of Nutrition and Dietetics.

Digital Object Identifier (DOI): doi:10.1016/j.jand.2014.05.005

Publisher's Link: http://www.sciencedirect.com/science/article/pii/S2212267214005012

This item was retrieved from the K-State Research Exchange (K-REx), the institutional repository of Kansas State University. K-REx is available at http://krex.ksu.edu 


\section{Position of the Academy of Nutrition and Dietetics: Nutrition and Lifestyle for a Healthy Pregnancy}

\section{Outcome}

\section{ABSTRACT}

It is the position of the Academy of Nutrition and Dietetics that women of childbearing age should adopt a lifestyle optimizing health and reducing risk of birth defects, suboptimal fetal development, and chronic health problems in both mother and child. Components leading to healthy pregnancy outcome include healthy prepregnancy weight, appropriate weight gain and physical activity during pregnancy, consumption of a wide variety of foods, appropriate vitamin and mineral supplementation, avoidance of alcohol and other harmful substances, and safe food handling. Pregnancy is a critical period during which maternal nutrition and lifestyle choices are major influences on mother and child health. Inadequate levels of key nutrients during crucial periods of fetal development may lead to reprogramming within fetal tissues, predisposing the infant to chronic conditions in later life. Improving the well-being of mothers, infants, and children is key to the health of the next generation. This position paper and the accompanying practice paper on the same topic provide registered dietitian nutritionists and dietetic technicians, registered; other professional associations; government agencies; industry; and the public with the Academy's stance on factors determined to influence healthy pregnancy, as well as an overview of best practices in nutrition and healthy lifestyles during pregnancy.

\section{POSITION STATEMENT}

It is the position of the Academy of Nutrition and Dietetics that women of childbearing age should adopt a lifestyle optimizing health and reducing risk of birth defects, suboptimal fetal development, and chronic health problems in both mother and child. Components leading to healthy pregnancy outcome include healthy prepregnancy weight, appropriate weight gain and physical activity during pregnancy, 
consumption of a wide variety of foods, appropriate vitamin and mineral supplementation, avoidance of alcohol and other harmful substances, and safe food handling.

This position paper provides Academy of Nutrition and Dietetics members, other professional associations, government agencies, industry, and the public with the Academy's stance on factors determined to influence healthy pregnancy, as well as emerging factors. Women with inappropriate weight gain, hyperemesis, multiple gestations, poor dietary patterns (eg, disordered eating), or chronic disease should be referred to a registered dietitian nutritionist (RDN) for medical nutrition therapy. For specific practice recommendations, refer to the Academy's practice paper on "Nutrition and Lifestyle for a Healthy Pregnancy Outcome." ${ }^{1}$

\section{TRENDS IMPACTING PREGNANCY OUTCOMES}

\section{Birth Defects, Low Birth Weight, Viable Birth Trends}

Pregnancy is a critical period during which maternal nutrition and lifestyle choices are major influences on mother and child health. Improving the well-being of mothers, infants, and children is key to the health of the next generation. One in 33 babies $(\cong 3 \%)$ is born with a birth defect; ${ }^{2}$ in 2010 , low birth weight (LBW) infants comprised $8.1 \%$ of US births. ${ }^{3}$ Birth defects and LBW are ranked $1^{\text {st }}$ and $2^{\text {nd }}$, respectively, among the 10 leading causes of death in US infants in $2006 .{ }^{3}$ A woman's chance of having a healthy baby improves when she adopts healthy behaviors, including good nutrition, recommended supplementation and avoidance of smoking, alcohol, and illicit drugs before becoming pregnant. ${ }^{2}$

\section{Obesity and Gestational Diabetes}


Prepregnancy body mass index (BMI) is an independent predictor of many adverse outcomes of pregnancy. The prevalence of obesity in women 12 to 44 years of age has more than doubled since 1976. In 1999-2004, nearly two-thirds of women of childbearing age were classified as overweight (BMI 225), and almost one-third were obese $(\mathrm{BMI} \geq 30){ }^{4}$ Overconsumption/overweight throughout the reproductive cycle are related to short- and long-term maternal health risks, including obesity, diabetes, dyslipidemia, and cardiovascular disease. Caloric excess does not guarantee adequate intake or nutrient status critical to healthy pregnancy outcomes. ${ }^{5}$

To improve maternal and child health outcomes, women should weigh within the normal BMI range when they conceive and strive to gain within ranges recommended by the Institute of Medicine (IOM) 2009 pregnancy weight guidelines. ${ }^{4}$ High rates of overweight and obesity are common in population subgroups already at risk for poor maternal and child health outcomes, compounding the need for intervention . ${ }^{4}$ In addition to health risks, gestational weight gain beyond the recommendation substantially increases risk of excess weight retention in obese women at 1 year postpartum. ${ }^{6}$ More information on obesity and pregnancy outcomes can be found in the "Position of the Academy of Nutrition and Dietetics and American Society for Nutrition: Obesity, Reproduction and Pregnancy Outcomes." 7

New diagnostic criteria for gestational diabetes mellitus (GDM) are expected to increase the proportion of women diagnosed with GDM, with potentially $18 \%$ of all pregnancies affected. ${ }^{8}$ Immediately following pregnancy, $5 \%$ to $10 \%$ of women with GDM are found to have diabetes, usually type 2 . Women who have had GDM have a $35 \%-60 \%$ chance of developing diabetes in the next $10-20$ years. ${ }^{8}$ RDNs can provide valuable guidance to women seeking assistance regarding optimal weight and healthy food selection before, during, and post-pregnancy. Additional information and guidance is available in the Academy's GDM Evidence-Based Nutrition Practice Guideline. ${ }^{9}$ 


\section{Hypertension and Preeclampsia}

Prevalence of chronic hypertension in pregnancy in the United States is estimated to be as high as 5\%. This is primarily attributable to the increased prevalence of obesity, as well as delay in childbearing to ages when chronic hypertension is more common. ${ }^{10}$ Hypertension in pregnancy can harm both mother and fetus, and women with chronic hypertension are more likely to experience preeclampsia ( $17 \%$ to $25 \%$ vs $3 \%$ to $5 \%$ in the general population). ${ }^{10}$ Age, preconception weight and health status, access to timely and appropriate health care, and poverty are some of the numerous factors affecting maternal health and the likelihood of a healthy pregnancy. Referral to the RDN and/or social worker may assure appropriate care will be available given the aforementioned factors that may influence maternal and fetal outcomes.

\section{OPTIMIZING PREGNANCY OUTCOMES WITH HEALTHY LIFESTYLE CHOICES}

Evidence is building that maternal diet and lifestyle choices influence the long-term health of the mother's children. Prepregnancy adherence to healthful dietary patterns, including the alternate Mediterranean Diet, Dietary Approaches to Stop Hypertension (DASH), and alternate Healthy Eating Index have been associated with a $24 \%$ to $46 \%$ lower risk of GDM. ${ }^{11}$ Population-based research provides evidence that maternal metabolic conditions may be associated with neurodevelopmental problems including autism and developmental delays in children. ${ }^{12}$ Inadequate levels of key nutrients during crucial periods of fetal development may lead to reprogramming within fetal tissues, predisposing the infant to chronic conditions in later life. Those conditions include obesity, cardiovascular disease, bone health, cognition, immune function, and diabetes. ${ }^{13}$

Maternal weight gain during pregnancy outside the recommended range is associated with increased risk to maternal and child health. ${ }^{4}$ While physiological responses to prenatal overnutrition result in poor health outcomes that emerge in childhood and adolescence, fetal undernutrition 
responses range from fetal survival to poor health outcomes emerging later in the offspring's adult life. ${ }^{14}$ The IOM recommends that more US women achieve gestational weight gain within the range identified for their prepregnant BMI. ${ }^{4}$ Pregnant women benefit from eating a variety of foods to meet nutrient needs and consuming sufficient calories to support recommended weight gain. Details regarding recommended energy requirements and recommended weight gain during pregnancy can be found in the related practice paper. ${ }^{1}$

\section{Energy Expenditure}

Physical activity during pregnancy benefits a woman's overall health. In a low-risk pregnancy, moderately-intense activity does not increase risk of LBW, preterm delivery, or miscarriage. ${ }^{16}$ Recreational moderate and vigorous physical activity during pregnancy is associated with a $48 \%$ lower risk of hyperglycemia, specifically among women with prepregnancy $\mathrm{BMI}<25 .{ }^{17} \mathrm{~A}$ prenatal nutrition and exercise program, regardless of exercise intensity, has been shown to reduce excessive gestational weight gain and decrease weight retention at 2 months post-partum in women of normal prepregnant BMI. $^{18}$

\section{Appropriate, Timely Nutrient Supplementation}

Iron. Iron deficiency with resultant anemia is the most prevalent micronutrient deficiency worldwide, affecting primarily pregnant or lactating women and young children. ${ }^{19}$ Iron deficiency anemia in pregnant women in industrialized countries is $17.4 \%,{ }^{20}$ with approximately $9 \%$ of adolescent girls and women of childbearing age in the United States having inadequate stores of body iron. ${ }^{19}$ The high incidence of iron deficiency underscores the need for iron supplementation in pregnancy. During the first two trimesters of pregnancy, iron deficiency anemia increases the risk for preterm labor, LBW, and 
infant mortality. ${ }^{21}$ Maternal and fetal demand for iron increases during pregnancy; this increase cannot be met without iron supplementation. ${ }^{19}$

Folic acid. Folic acid is recognized as important prior to and during pregnancy because of its preventive properties against neural tube defects. All women, including adolescents, who are capable of becoming pregnant should consume 400 mcg per day of folic acid from fortified foods and/or dietary supplements, in addition to eating food sources of folate. ${ }^{15}$ Pregnant women are advised to consume 600 mcg dietary folate equivalents daily from all food sources. Dietary folate equivalents adjust for the difference in bioavailability of food folate compared with synthetic folic acid. One dietary folate equivalent is equal to $1 \mathrm{mcg}$ food folate, which is equal to $0.6 \mathrm{mcg}$ folic acid derived from supplements and fortified foods taken with meals. ${ }^{14}$ Women who have had an infant with a neural tube defect should consult with their health care provider regarding the recommendation to take $4,000 \mathrm{mcg}$ folic acid daily prior to and throughout the first trimester of pregnancy. ${ }^{22}$ An association between the lack of periconceptual use of vitamins or supplements containing folic acid with an excess risk for birth defects due to diabetes mellitus ${ }^{23}$ highlights ongoing research.

Vitamin D. The function of vitamin D during pregnancy for both mother and fetus is not fully defined at present. ${ }^{24}$ Though vitamin D supplementation during pregnancy has been suggested as an intervention to protect against adverse gestational outcomes including LBW, ${ }^{25}$ the need, safety, and effectiveness of vitamin D supplementation during pregnancy remains controversial. ${ }^{24}$ The IOM recommends $600 \mathrm{IU}$ per day of vitamin $D$ to meet the needs of most North American adults, including pregnant women. ${ }^{26}$ Ongoing research suggests higher levels of supplementation are safe and effective in improving maternal and infant vitamin D status. ${ }^{24}$ 
Choline. Choline is an essential nutrient during pregnancy, due to its high rate of transport from mother to fetus. Maternal deficiency of choline can interfere with normal fetal brain development. While choline is found in many foods, the majority of pregnant women are not achieving the Adequate Intake for pregnancy of $450 \mathrm{mg}$ choline per day. ${ }^{27}$

Calcium. The Dietary Reference Intake for calcium in pregnancy is equal to that of non-pregnant women of the same age ${ }^{26}$ due to increased efficiency in calcium absorption during pregnancy and maternal bone calcium mobilization. ${ }^{26}$ Women with suboptimal intakes ( $<500 \mathrm{mg} /$ day) may need additional amounts to meet both maternal and fetal bone requirements. ${ }^{28}$

lodine. lodine is required for normal brain development and growth; iodine deficiency worldwide is a growing concern. During pregnancy, iodine requirements increase, making mother and developing fetus vulnerable. Congenital hypothyroidism is associated with cretinism, while clinical hypothyroidism has been associated with increased risk of poor perinatal outcomes including prematurity, LBW, miscarriage, preeclampsia, fetal death, and impaired fetal neurocognitive development. ${ }^{29}$ Recent national surveys indicate a subset of pregnant and lactating US women may have mild to moderately inadequate dietary iodine intake. ${ }^{30}$ The IOM recommends an iodine intake from dietary and supplement sources of $150 \mathrm{mcg}$ per day pre-conception, and 220 mcg per day for pregnant women. ${ }^{31}$

\section{Environmental and Dietary Issues}

Foodborne illness during pregnancy. Pregnant women and their fetuses are at increased risk of developing foodborne illness due to hormonal changes of pregnancy that lead to decreased cellmediated immune function. Of greatest concern during pregnancy are Listeria monocytogenes, Toxoplasma gondii, Brucella species, Salmonella species, and Campylobacter jejuni. ${ }^{30}$ Pregnant women 
should closely adhere to food safety recommendations outlined in the Dietary Guidelines for Americans. ${ }^{14}$ Updated food safety guidelines can be reviewed on the Food and Drug Administration at www.fda.gov/Food/ResourcesForYou/HealthEducators/ucm083308.htm.

Benefits/concerns regarding fish/seafood consumption. The nutritional value of seafood is particularly important during fetal growth and development, as well as in early infancy and childhood. ${ }^{14}$ Intake of n-3 fatty acids, particularly docosahexaenoic acid, from at least 8 oz of seafood per week for pregnant women is associated with improved infant visual and cognitive development. ${ }^{14}$ While prenatal mercury exposure (1 mcg/g or greater) was found to be associated with a greater risk of attention-deficit hyperactivity disorder-related behaviors, prenatal fish consumption of more than two servings per week was protective of those behaviors. ${ }^{33}$ RDNs and dietetic technicians, regsitered can help pregnant women balance the benefits of eating fish while avoiding high-mercury content seafood.

Non-nutritive sweeteners. While calorie and blood glucose control are acknowledged benefits of nonnutritive sweeteners, limited research addresses the safety of non-nutritive sweeteners on healthy pregnancy or in GDM. ${ }^{34}$

Alcohol. Alcohol should not be consumed by pregnant women or those who may become pregnant. ${ }^{14}$ Drinking alcohol during pregnancy, especially in early pregnancy, may result in behavioral or neurological defects in the offspring and affect a child's future intelligence. No safe level of alcohol consumption during pregnancy has been established. ${ }^{14}$

Caffeine. Caffeine half-life increases in pregnancy from 3 hours in the first trimester to 80-100 hours in late pregnancy. Women who are pregnant or trying to become pregnant are advised by the American 
Council of Obstetricians and Gynecologists ${ }^{35}$ to consume no more than $200 \mathrm{mg}$ of caffeine per day - the approximate amount in one 12-oz cup of coffee. However, birth defects research indicates moderate or high amounts of beverages and foods containing caffeine do not increase the risk of congenital malformations, miscarriage, preterm birth, or growth retardation. ${ }^{36}$

Hydration and water needs. Adequate hydration is essential to healthy pregnancy, as a woman accumulates 6 to 9 liters of water during gestation. The total water Adequate Intake for pregnancy

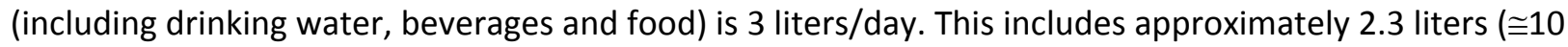
cups) as total beverages. ${ }^{37}$

Energy drinks. An energy drink is any beverage that contains some form of legal stimulant and/or vitamins added to provide a short-term boost in energy. These drinks may contain substantial and varying amounts of sugar and caffeine, as well as taurine, carnitine, inositol, ginkgo, and milk thistle. Many of these have not been studied for safety during pregnancy. Ginseng, another common ingredient, is not recommended for use during pregnancy. The avoidance of energy drinks during pregnancy is advised.

Sugar-sweetened drinks. Sugar-sweetened beverages, including regular sodas, sports drinks, energy drinks, and fruit drinks, provide $35.7 \%$ of added sugars in the US diet. ${ }^{14}$ Reduced consumption of sources of added sugars lowers the calorie content of the diet without compromising nutrient adequacy.

\section{Health Conditions Between and After Pregnancies}

Maternal return to healthy weight status postpartum can prevent future overweight and obesity.${ }^{14}$ The Dietary Guidelines for Americans forms the basis for nutrition counseling for postpartum women, and 
RDNs and dietetic technicians, registered can assist women in achieving their prepregnancy weight.

Outside of weight status, recent research has shown that diet quality, dietary intake, and overall nutritional status can affect the risk of postpartum depression. An association between $\mathrm{n}-3$ fatty acids, serotonin transporter genotype, and postpartum depression has been identified. ${ }^{38}$ Low-income women with depressive symptoms and life stressors are at risk for low-prenatal diet quality, so intensive dietary intervention before and during pregnancy may be needed to promote optimal health. ${ }^{39}$ The risk of maternal and infant mortality and pregnancy-related complications can be reduced with increased access to quality interconception care.

\section{CONCLUSIONS}

Pregnancy has been regarded as a maternal phase with requisite additional nutritional requirements; mounting evidence suggests that the prenatal period constitutes a critical convergence of short- and long-term factors affecting the lifelong health of mother and child. The aim of prenatal nutrition is to support a healthy uterine environment for optimal fetal development while supporting maternal health. ${ }^{5}$ The ideal prenatal diet should limit overconsumption for the mother and prevent undernutrition for the fetus; ${ }^{5}$ a healthy lifestyle includes regular physical activity and avoidance of harmful practices.

\section{References}

1. Academy of Nutrition and Dietetics. Practice Paper of the Academy of Nutrition and Dietetics: Nutrition and lifestyle for a healthy pregnancy outcome. http://www.eatright.org/members/practicepapers/. Published July 1, 2014.

2. Centers for Disease Control and Prevention. Division of Birth Defects and Developmental Disabilities. Birth defects. http://www.cdc.gov/ncbddd/birthdefects/index.html. Accessed October 4, 2012.

3. Centers for Disease Control and Prevention. FastStats: births and natality. http://www.cdc.gov/nchs/fastats/births.htm. Accessed October 4, 2012.

4. Committee to Re-examine the Institute of Medicine Pregnancy Weight Guidelines. Weight Gain During Pregnancy: Reexamining the Guidelines. In: Rasmussen KM, Yaktine AL, eds. Washington, 
DC: The National Academies Press; 2009. IOM (Institute of Medicine) and NRC (National Research Council). 2009. Weight gain during pregnancy: Reexamining the guidelines. Washington, DC: The National Academies Press.

5. Shapira N. Prenatal nutrition: A critical window of opportunity for mother and child. Womens Health. 2008;4(6):639-656.

6. Vesco KK, Dietz PM, Rizzo J, et al. Excessive gestational weight gain and postpartum weight retention among obese women. Obstet Gynecol. 2009;114(5)1069-1075.

7. Academy of Nutrition and Dietetics. Position of the Academy of Nutrition and Dietetics and American Society for Nutrition: Obesity, reproduction and pregnancy outcomes. J Am Diet Assoc. 2009;109(5):918-927.

8. Centers for Disease Control and Prevention. 2011 National Diabetes fact sheet: gestational diabetes in the United States. http://www.cdc.gov/diabetes/pubs/estimates11.htm. Accessed November 11, 2012.

9. Academy of Nutrition and Dietetics Evidence Analysis Library. Gestational diabetes evidencebased nutrition practice guideline. http://andevidencelibrary.com/topic.cfm?cat=3733. Accessed December 6, 2013.

10. Seely EW, Ecker J. Chronic hypertension in pregnancy. N Engl J Med. 2011;365(5):439-436.

11. Tobias DK, Zhang C, Chavarro J, et al. Prepregnancy adherence to dietary patterns and lower risk of gestational diabetes. Am J Clin Nutr. 2012;96(2):289-295.

12. Krakowiak $P$, Walker CK, Bremer AA, et al. Maternal metabolic conditions and risk for autism and other neurodevelopmental disorders. Pediatrics. 2012;129(5):e1121-e1128.

13. Hanley B, Dijane J, Fewtrell M, et al. Metabolic imprinting, programming and epigenetics - a review of present priorities and future opportunities. Br J Nutr. 2010;104(suppl 1):S1-S25.

14. McMillen IC, MacLaughlin SM, Muhlhausler BS, Gentili S, Duffield JL, Morrison JL. Developmental origins of adult health and disease: The role of periconceptional and foetal nutrition. Basic Clin Pharmacol Toxicol. 2008;102(2):82-89.

15. US Department of Agriculture, US Department of Health and Human Services. Dietary Guidelines for Americans, 2010. $7^{\text {th }}$ ed. Washington, DC: US Government Printing Office; 2010.

16. US Department of Health and Human Services. Physical activity for women during pregnancy and the postpartum period. In: 2008 Physical Activity Guidelines for Americans. Washington, DC: Office of Disease Prevention \& Health Promotion. http://www.health.gov/paguidelines/guidelines/default.aspx. Accessed September 25, 2012.

17. Deierlein AL, Siega-Riz AM, Evenson KR. Physical activity during pregnancy and risk of hyperglycemia. J Womens Health. 2012;21(7):769-775.

18. Ruchat SM, Davenport MH, Giroux I, et al. Nutrition and exercise reduce excessive weight gain in normal-weight pregnant women. Med Sci Sports Exer.c 2012;44(8):1419-1426.

19. Khalafallah AA, Dennis AE. Iron deficiency anaemia in pregnancy and postpartum: Pathophysiology and effect of oral versus intravenous iron therapy. J Pregnancy. 2012; Article ID 630519.

20. US Department of Agriculture, US Department of Health and Human Services. 2010 US Dietary Guidelines Advisory Committee. Part D. Section 2: Nutrient adequacy. In: Report of the Dietary Guidelines Advisory Committee on the Dietary Guidelines for Americans, 2010. $7^{\text {th }}$ Edition, Washington, DC: U.S. Government Printin Office, December 2010.

21. Gautam CS, Saha L, Sekhri K, and Saha PK. Iron deficiency in pregnancy and the rationality of iron supplements prescribed during pregnancy. Medscape J Med. 2008;10(12):283-288. http://www.ncbi.nlm.nih.gov/pmc/articles/PMC264404/. Accessed October 3, 2012.

22. Centers for Disease Control and Prevention. Folic acid: recommendations. http://www.cdc.gov/ncbddd/folicacid/recommendations.html. Accessed September 24, 2012. 
23. Correa A, Gilboa SM, Botto LD, et al. Lack of periconceptional vitamins or supplements that contain folic acid and diabetes mellitus-associated birth defects. Am J Obstet Gynecol. 2012;206(3):218.e1-e13.

24. Hollis BW, Johnson D, Hulsey TC, Ebeling M, Wagner CL. Vitamin D supplementation during pregnancy: Double-blind, randomized clinical trial of safety and effectiveness. J Bone Miner Res. 2011;26(10):2341-2357.

25. Thorne-Lyman A, Fawzi WW. Vitamin D during pregnancy and maternal, neonatal and infant health outcomes: A systematic review and meta-analysis. Paediatr Perinat Epidemiol.2012;26(suppl 1):75-90.

26. Institute of Medicine. Dietary Reference Intakes for calcium and vitamin D.2010. http://www.iom.edu/Reports/2010/Dietary-Reference-Intakes-for-Calcium-and-Vitamin-D.aspx. Published November 30, 2010. Accessed September 4, 2012.

27. Caudill MA. Pre- and postnatal health: Evidence of increased choline needs. J Am Diet Assoc. 2010;110(8):1198-1206.

28. Hacker AN, Fung EB, King JC. Role of calcium during pregnancy: maternal and fetal needs. Nutr Rev. 2012;70(7):397-409.

29. Obican SG, Jahnke GD, Soldin OP, Scialli AR. Teratology public affairs committee position paper: lodine deficiency in pregnancy. Birth Defects Res. 2012;94(part A):677-682.

30. Stagnaro-Green A, Abalovich M, Alexander E, et al. Guidelines of the American Thyroid Association for the diagnosis and management of thyroid disease during pregnancy and postpartum. Thyroid. 2011;21(10):1081-1125.

31. Swanson C, Zimmermann M, Skeaff S, et al. Summary of an NIH workshop to identify research needs to improve the monitoring of iodine status in the United States and to inform the DRI. J Nutr. 2012;142(6):1175S-1185S.

32. Dean J, Kendall P. Food safety during pregnancy. 2012;9.372. Colorado State University Extension. Food and Nutrition Series. http://www.ext.colostate.edu/pubs/foodnut/09372.pdf. Accessed December 5, 2012.

33. Sagiv SK, Thurston SW, Bellinger DC, Amarasiriwardena C, Korrick SA. Prenatal exposure to mercury and fish consumption during pregnancy and attention-deficit/hyperactivity disorderrelated behavior in children. Arch Pediatr Adolesc Med. Doi:10.1001/archpediatrics.2012.1286. Accessed October 21, 2012.

34. Academy of Nutrition and Dietetics Evidence Analysis Library. Pregnancy and nutrition - nonnutritive sweeteners. http://andevidencelibrary.com/evidence.cfm?evidence summary id=250587 Accessed December 3, 2013.

35. American College of Obstetrics and Gynecology. Committee opinion no. 462: Moderate caffeine consumption during pregnancy. Obstet Gynecol. 2010;116(2,pt 1):467-468.

36. Brent RL, Christian MS, Diener RM. Evaluation of the reproductive and developmental risks of caffeine. Birth Defects Res (Part B). 2011;92(2):152-187.

37. Institute of Medicine. Dietary reference intakes for water, potassium, sodium, chloride, and sulfate. http://www.nap.edu/openbook.php?record id=10925\&page=151. Published 2005. Accessed October 21, 2012.

38. Shapiro GD, Fraser WD, Séguin JR. Emerging risk factors for postpartum depression: Serotonin transporter genotype and omega-3 fatty acid status. Can J Psychiatry. 2012;57(11):704-712.

39. Fowles ER, Stang J, Bryant M, Kim SH. Stress, depression, social support, and eating habits reduce diet quality in the first trimester in low-income women: A pilot study. J Acad Nutr Diet. 2012;112(10):1619-1625. 